\title{
Experimental validation of a new adaptive control scheme for quadrotors MAVs
}

\author{
Gianluca Antonelli ${ }^{\dagger}$, Elisabetta Cataldi ${ }^{\dagger}$, Paolo Robuffo Giordano ${ }^{\ddagger}$, Stefano Chiaverini $^{\dagger}$, Antonio Franchi ${ }^{2}$
}

\begin{abstract}
In this paper, an adaptive trajectory tracking controller for quadrotor MAVs is presented. The controller exploits the common assumption of a faster orientation dynamics w.r.t. the translational one, and is able to asymptotically compensate for parametric uncertainties (e.g., displaced center of mass), as well as external disturbances (e.g., wind). The good performance of the proposed controller is then demonstrated by means of an extensive experimental evaluation performed with a commercially-available quadrotor MAV.
\end{abstract}

\section{INTRODUCTION}

Over the last years the robotics community experienced a substantial increase of interest in the Micro Aerial Vehicles (MAVs) field. Among the numerous tasks attainable with MAV systems, one can list aerial mapping, air pollution monitoring, traffic management, inspection of damaged buildings and dangerous sites, and many agricultural applications such as pesticide spraying. Furthermore, commercial services based on MAV technology recently appeared in the market [1]. In fact, when compared to traditional fixed-wing aircrafts, MAVs have several advantages, such as the ability to take-off and land vertically, to hover above any target, and to navigate in indoor or highly cluttered environments.

Development of effective flight controllers and motion planning strategies has been one of the primary objectives in MAV research, see e.g. [2], [3]. In most of these works, the experimental validation has been performed by exploiting onboard IMUs and external motion capture systems in order to obtain an accurate and fast estimation of the MAV state. In parallel, another fundamental research topic has been addressed over the last years: the estimation of the MAV position/orientation by only resorting to onboard sensors and computation capabilities, see, e.g., [4], [5], [6]. MAVs have also been proven useful for many tasks involving physical interaction such as cooperative transportation [7] and aerial grasping [8]. Cooperative sensing and multi-MAV coordination have also been addressed in the literature [9], [10], [11], [12].

Quadrotors, i.e., MAVs equipped with four aligned coplanar propellers, have been the most popular MAVs in the last decade. In the overview offered by [13], [14] the mechanical model of the quadrotor is derived assuming perfect geometrical inertial symmetry. This assumption allows for a simpler design of the controllers, such as, for instance, by

$\dagger$ G. Antonelli, E. Cataldi and S. Chiaverini are with University of Cassino and Southern Lazio, Cassino, Italy \{antonelli, chiaverini\}@unicas.it

$\ddagger$ P. Robuffo Giordano is with the CNRS at IRISA, Rennes Cedex, France prgeirisa.fr

2 A. Franchi is with the Max Planck Institute for Biological Cybernetics, Tübingen, Germany antonio.franchi@tuebingen.mpg.de using LQR techniques. Additional control strategies based on sliding mode and backstepping techniques, and still exploiting the above-mentioned assumption, have been also presented [15], [16]. Being the quadrotor an underactuated mechanical system (only four control inputs are available despite the six dimensions of its configuration), a common strategy is to control its 3D position and yaw angle, i.e., quantities know to be flat outputs for the system [17]. The addition of tilting propellers to the classical quadrotor model has nevertheless been recently explored in [18] in order to increase actuation capabilities.

Robustness of the flight controller performance is a fundamental feature for any MAV application. Integral-based actions can be used to counteract external disturbances, such as wind and presence of small loads. Nevertheless, an adaptive/integral action may result in an additional disturbance when the nonlinearities of the model are not properly taken into account, see, e.g., [19] for analogous problems in the marine context. In this sense, at the best of our knowledge the only adaptive control for MAVs has been proposed in [20], [21]. This solution employes a feedback linearization approach which exploits the linear dependency of the model w.r.t. the position of the Center of Mass (CoM).

In [22] a novel adaptive control scheme for a quadrotor that considers the effect of constant exogenous forces and moments, and allows for presence of unknown dynamic parameters (e.g., the position of the CoM) has been introduced and its theoretical analysis provided. The standard assumption of a rotational dynamics faster than the positional is assumed and, contrarily to [20], the external disturbances are explicitly considered in the design. A minimal set of parameters for the identification and the possible wrong estimation of the center of mass have been considered. Here an experimental validation of the proposed method on a real MAV platform is presented.

\section{MATHEMATICAL BACKGROUND}

\section{A. Kinematics}

A rigid body is completely described by its position and orientation with respect to a reference frame $\Sigma_{i}, O-\boldsymbol{x y} \boldsymbol{z}$ assumed earth-fixed and inertial. Let $\boldsymbol{\eta}_{1}=\left[\begin{array}{lll}x & y & z\end{array}\right]^{\mathrm{T}} \in$ $\mathbb{R}^{3}$ be the vector of the body position coordinates in an earthfixed reference frame. The vector $\dot{\eta}_{1}$ is the corresponding time derivative (expressed in the earth-fixed frame). If one defines $\boldsymbol{\nu}_{1}=\left[\begin{array}{lll}u & v & w\end{array}\right]^{\mathrm{T}}$ as the linear velocity of the origin of the body-fixed frame $\Sigma_{b}, O_{b}-\boldsymbol{x}_{b} \boldsymbol{y}_{b} \boldsymbol{z}_{b}$ with respect to the origin of the earth-fixed frame, expressed in the bodyfixed frame (from now on: body-fixed linear velocity), then 
the following relation between the defined linear velocities holds:

$$
\boldsymbol{\nu}_{1}=\boldsymbol{R}_{I}^{B} \dot{\boldsymbol{\eta}}_{1}
$$

where $\boldsymbol{R}_{I}^{B}$ is the rotation matrix expressing the transformation from the inertial frame to the body-fixed frame [23].

Define $\boldsymbol{\eta}_{2}=\left[\begin{array}{lll}\varphi & \theta & \psi\end{array}\right]^{\mathrm{T}} \in \mathbb{R}^{3}$ as the set of body Eulerangle coordinates with respect to the earth-fixed reference frame. Those are commonly named roll, pitch and yaw angles and correspond to the succesive elementary rotations around $x, y$ and $z$ in the fixed frame [23]. Let define $\boldsymbol{\nu}_{2}=$ $\left[\begin{array}{lll}p & q & r\end{array}\right]^{\mathrm{T}}$ as the angular velocity of the body-fixed frame with respect to the earth-fixed frame expressed in the bodyfixed frame (from now on: body-fixed angular velocity). Finally $\boldsymbol{\nu}=\left[\begin{array}{ll}\boldsymbol{\nu}_{1}^{\mathrm{T}} & \boldsymbol{\nu}_{2}^{\mathrm{T}}\end{array}\right]^{\mathrm{T}} \in \mathbb{R}^{6}$ is the six-dimensional bodyfixed velocity.

\section{B. Dynamics}

The rigid body dynamics of a quadrotor, in matrix form, is given by:

$$
\boldsymbol{M} \dot{\boldsymbol{\nu}}+\boldsymbol{C}(\boldsymbol{\nu}) \boldsymbol{\nu}+\boldsymbol{\tau}_{v, W}+\boldsymbol{g}\left(\boldsymbol{R}_{I}^{B}\right)=\boldsymbol{\tau}_{v},
$$

where $\boldsymbol{\tau}_{v}=\left[\begin{array}{ll}\boldsymbol{\tau}_{1}^{\mathrm{T}} & \boldsymbol{\tau}_{2}^{\mathrm{T}}\end{array}\right]^{\mathrm{T}}$, the vector

$$
\boldsymbol{\tau}_{1}=\left[\begin{array}{lll}
X & Y & Z
\end{array}\right]^{\mathrm{T}},
$$

collects the linear forces acting on the rigid body expressed in a body-fixed frame, while

$$
\boldsymbol{\tau}_{2}=\left[\begin{array}{lll}
K & M & N
\end{array}\right]^{\mathrm{T}}
$$

collects the moments acting on the rigid body expressed in a body-fixed frame. Deatils on the terms can be found, e.g., in [23], [24]. Notice that the term $\boldsymbol{\tau}_{v, W} \in \mathbb{R}^{6}$ represents external disturbances such as wind; its effect on the vehicle is modeled as a constant disturbance in the earth-fixed frame that is further projected onto the vehicle-fixed frame [22].

It is possible to rewrite eq. (2) by exploiting the linearity in the parameters as:

$$
\boldsymbol{\Phi}_{v}\left(\dot{\nu}, \nu, \boldsymbol{R}_{I}^{B}\right) \gamma_{v}=\boldsymbol{\tau}_{v}
$$

where $\gamma_{v} \in \mathbb{R}^{16}$ is the vector of the dynamic parameters collecting the mass ( 1 parameter), the first moment of inertia (3 parameters), the inertia tensor (6 parameters) and the 6 elements of the disturbance $\gamma_{v, W}$. The same equation may be easily rewritten with respect to the variables expressed in the inertial frame $\boldsymbol{\eta}, \dot{\boldsymbol{\eta}}, \ddot{\boldsymbol{\eta}}$ following the guidelines of, e.g., [23]. In the following, the terms $\boldsymbol{\Phi}_{x y} \in \mathbb{R}^{2 \times 16}$ and $\phi_{z} \in \mathbb{R}^{1 \times 16}$ will denote the first two rows and third row of the regressor matrix expressed in the inertial frame, respectively. Following the guidelines of [25], well established in robotics [26], it is possible to further elaborate the regressor and classify the parameters among the sets: unidentifiable, identifiable alone and identifiable in linear combination. As an example, the body is affected by a vertical force caused by both the gravity and the wind; those effects cannot be separated and the corresponding parameters will be identifiable only in a linear combination. For sake of space, in this work the details are omitted; the controller tested in the following, in fact, will only consider the parameters that affect the steady state error.

\section{Thrust}

Quadrotors are equipped with 4 thrusters aligned along the body-fixed $z$ axis with position $\boldsymbol{p}_{t, i}^{b} \in \mathbb{R}^{3}$, each of them providing a force and a moment

$$
f_{i}=b \omega_{t, i}^{2} \quad \tau_{t, i}=d \omega_{t, i}^{2} \quad \text { for } i=1, \ldots, 4
$$

where $\omega_{t, i}$ is the angular velocity of the $i$ th rotor, $b$ and $d$ are the thrust and drag coefficients. Figure 1 reports the common motor position with relevant variables. Notice that the body-fixed frame is positioned in the geometric center of the vehicle, i.e., in the intersection between the two thrusters axes.

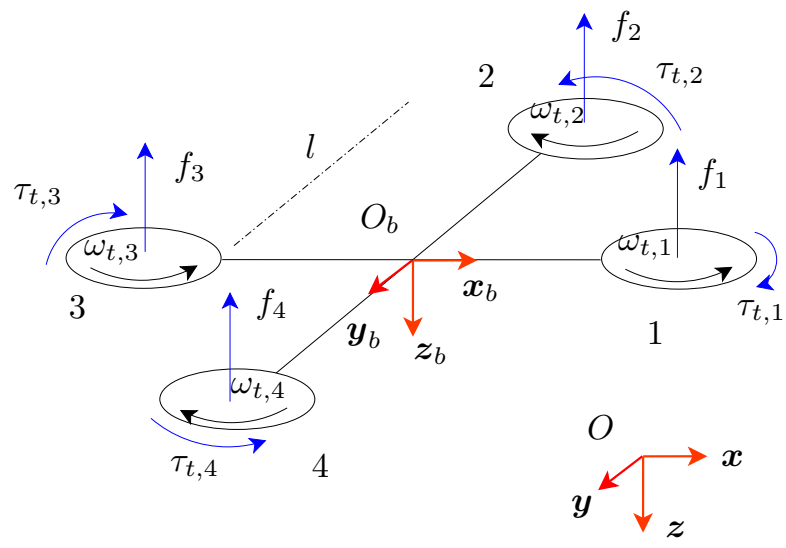

Fig. 1. Thrusters related variables

It holds

$$
\boldsymbol{\tau}_{1}=\left[\begin{array}{lll}
0 & 0 & \sum_{i=1}^{4} f_{i}
\end{array}\right]^{\mathrm{T}}
$$

and, by defining $\boldsymbol{r}_{C}^{b}=\left[\begin{array}{lll}r_{C, x} & r_{C, y} & r_{C, z}\end{array}\right]^{\mathrm{T}} \neq \mathbf{0}$, it is possible to compute:

$$
\boldsymbol{\tau}_{2}=\left[\begin{array}{c}
l\left(f_{2}-f_{4}\right)+r_{C, y}\left(f_{2}+f_{4}\right) \\
l\left(f_{1}-f_{3}\right)+r_{C, x}\left(f_{1}+f_{3}\right) \\
-\tau_{t, 1}+\tau_{t, 2}-\tau_{t, 3}+\tau_{t, 4}
\end{array}\right]
$$

It is finally possible to write the mapping from the angular velocities to the force-torque at the vehicle:

$$
\left[\begin{array}{l}
Z \\
K \\
M \\
N
\end{array}\right]=\boldsymbol{B}_{v}\left[\begin{array}{l}
\omega_{t, 1}^{2} \\
\omega_{t, 2}^{2} \\
\omega_{t, 3}^{2} \\
\omega_{t, 4}^{2}
\end{array}\right]
$$

with

$$
\boldsymbol{B}_{v}=\left[\begin{array}{cccc}
b & b & b & b \\
0 & b\left(l+r_{C, y}\right) & 0 & -b\left(l-r_{C, y}\right) \\
b\left(l+r_{C, x}\right) & 0 & -b\left(l-r_{C, x}\right) & 0 \\
-d & d & -d & d
\end{array}\right] \text {. }
$$

\section{QUADROTOR ADAPTIVE CONTROL}

The thrusters velocities may be assumed as the control input for the quadrotor control problem. The dynamics of the low level motor controller, in fact, can be typically neglected with respect to the vehicle dynamics; thus, we can 
assume $\boldsymbol{u}=\left[\begin{array}{llll}\omega_{t, 1}^{2} & \omega_{t, 2}^{2} & \omega_{t, 3}^{2} & \omega_{t, 4}^{2}\end{array}\right]^{\mathrm{T}}$. Since the controller will output the desired force $\left[\begin{array}{llll}Z_{c} & K_{c} & M_{c} & N_{c}\end{array}\right]^{\mathrm{T}}$ at the vehicle, the control input is obtained as

$$
\boldsymbol{u}=\boldsymbol{B}_{v}^{-1}\left[\begin{array}{c}
Z_{c} \\
K_{c} \\
M_{c} \\
N_{c}
\end{array}\right]
$$

where $\boldsymbol{B}_{v}^{-1} \in \mathbb{R}^{4 \times 4}$ is the inverse of (4).

It is interesting to evaluate what happens if the mapping from the desired forces to the thrusters velocities is computed with the estimated mapping $\left(\hat{\boldsymbol{r}}_{C}\right)$, while the effective mapping is physically related to $\boldsymbol{r}_{C}$ :

$$
\left[\begin{array}{c}
Z \\
K \\
M \\
N
\end{array}\right]=\left.\left.\boldsymbol{B}_{v}\right|_{\boldsymbol{r}_{C}} \boldsymbol{B}_{v}^{-1}\right|_{\hat{\boldsymbol{r}}_{C}}\left[\begin{array}{c}
Z_{c} \\
K_{c} \\
M_{c} \\
N_{c}
\end{array}\right]
$$

i.e.,

$$
\left[\begin{array}{c}
Z \\
K \\
M \\
N
\end{array}\right]=\left[\begin{array}{cccc}
1 & 0 & 0 & 0 \\
\frac{\tilde{r}_{C, y}}{2} & 1 & 0 & \frac{b \tilde{r}_{C, y}}{2 d} \\
\frac{\tilde{r}_{C, x}}{2} & 0 & 1 & -\frac{b \tilde{r}_{C, x}}{2 d} \\
0 & 0 & 0 & 1
\end{array}\right]\left[\begin{array}{c}
Z_{c} \\
K_{c} \\
M_{c} \\
N_{c}
\end{array}\right]
$$

where ? represents the error operator and the terms non belonging to the unitary matrix represent a coupling effect that may arise if the center of mass is wrongly estimated or neglected. Also, force along $\boldsymbol{z}_{b}$ and moment around $\boldsymbol{z}_{b}$ are not affected by a wrong estimation of the center of mass and thus $Z=Z_{c}$ and $N=N_{c}$.

In the following, an adaptive control law for quadrotor position and yaw regulation will be developed by taking into account persistent external disturbances and unknown CoM position. The assumption that the roll and pitch dynamics are faster than the position one is made. Figure 2 sketches the control loop.

\section{A. Altitude control}

Let define $\tilde{z}=z_{d}-z \in \mathbb{R}, s_{z}=\dot{\tilde{z}}+\lambda_{z} \tilde{z} \in \mathbb{R}$ with $\lambda_{z}>0$ and $\tilde{\gamma}_{v}=\gamma_{v}-\hat{\gamma}_{v}$ with the hat symbol denoting the estimate of the corresponding variable. The altitude controller is given by:

$$
\begin{aligned}
Z & =\frac{1}{\cos \varphi \cos \theta}\left(\phi_{z} \hat{\gamma}_{v}+k_{p z} s_{z}\right) \\
\dot{\hat{\gamma}}_{v} & =\boldsymbol{K}_{\gamma, z}^{-1} \boldsymbol{\phi}_{z}^{\mathrm{T}} s_{z}
\end{aligned}
$$

in which $\phi_{z}=\phi_{z}\left(\ddot{z}_{d}+\lambda_{z} \dot{\tilde{z}}, \dot{\boldsymbol{\eta}}, \boldsymbol{R}_{I}^{B}\right), \boldsymbol{K}_{\gamma, z}^{-1}>\boldsymbol{O}$, and $k_{p z}>$ 0 .

It is interesting to implement a simpler version of the controller aimed at compensating the sole persistent dynamic terms, i.e., those terms preventing a null steady state error, yielding:

$$
\begin{aligned}
Z & =\frac{1}{\cos \varphi \cos \theta}\left(\hat{\gamma}_{z}+k_{p z} s_{z}\right) \\
\dot{\hat{\gamma}}_{z} & =k_{\gamma, z}^{-1} s_{z}
\end{aligned}
$$

in which $\gamma_{z} \in \mathbb{R}$ embeds the joint contribution of the gravity and the vertical wind effects.

\section{B. Horizontal position control}

Let us recall the elementary rotation around $z$ as

$$
\boldsymbol{R}_{z}=\left[\begin{array}{cc}
\cos (\psi) & \sin (\psi) \\
-\sin (\psi) & \cos (\psi)
\end{array}\right]
$$

and define as $\tilde{\boldsymbol{\eta}}_{x y}=\left[\begin{array}{ll}x_{d}-x & y_{d}-y\end{array}\right]^{\mathrm{T}} \in \mathbb{R}^{2}$ and $\boldsymbol{s}_{x y}=$ $\dot{\tilde{\boldsymbol{\eta}}}_{x y}+\lambda_{x y} \tilde{\boldsymbol{\eta}}_{x y} \in \mathbb{R}^{2}$ with $\lambda_{x y}>0$. The virtual inputs $\varphi$ and $\theta$ are selected as the solutions of:

$$
\begin{aligned}
{\left[\begin{array}{c}
c_{\varphi} s_{\theta} \\
-s_{\varphi}
\end{array}\right] } & =\frac{1}{Z} \boldsymbol{R}_{z}\left(\boldsymbol{\Phi}_{x y} \hat{\gamma}_{v}+k_{p, x y} \boldsymbol{s}_{x y}\right) \\
\dot{\hat{\gamma}}_{v} & =\boldsymbol{K}_{\gamma, x y}^{-1} \boldsymbol{\Phi}_{x y}^{\mathrm{T}} \boldsymbol{s}_{x y} .
\end{aligned}
$$

with $\boldsymbol{\Phi}_{x y}=\boldsymbol{\Phi}_{x y}\left(\ddot{\boldsymbol{\eta}}_{d, x y}+\lambda_{x y} \dot{\tilde{\boldsymbol{\eta}}}_{x y}, \dot{\boldsymbol{\eta}}, \boldsymbol{R}_{I}^{B}\right) \in \mathbb{R}^{2 \times 16}$.

Also in this case, by only considering the persistent dynamic terms, the controller reduces to a simple

$$
\begin{aligned}
{\left[\begin{array}{c}
c_{\varphi} s_{\theta} \\
-s_{\varphi}
\end{array}\right] } & =\frac{1}{Z} \boldsymbol{R}_{z}\left(\hat{\gamma}_{x y}+k_{p, x y} \boldsymbol{s}_{x y}\right) \\
\dot{\hat{\gamma}}_{x y} & =k_{\gamma, x y}^{-1} \boldsymbol{s}_{x y}
\end{aligned}
$$

where $\hat{\gamma}_{x y} \in \mathbb{R}^{2}$ represents the sole wind effect supposed constant in the inertial frame, and $\varphi$ and $\theta$ can be easily computed yielding the desired $\varphi_{d}$ and $\theta_{d}$ sent to the orientation controller.

\section{Orientation control}

The orientation control receives as input the desired roll, pitch and yaw; the formers are obtained by the position control equations. Notice that, in this case, it is necessary to explicitly consider the presence of a coupling effect among the desired and obtained forces as shown in eqs. (6)-(7). It is worth noticing that neither the altitude nor the yaw control loop are affected by $\tilde{\boldsymbol{r}}_{C}$. The convergence to a steady state value for $Z_{c}$ and $N_{c}$ can thus be assumed. In any case, roll and pitch control can be designed by considering the estimation error as an external, constant, disturbance:

$$
\begin{aligned}
K & =K_{c}+\frac{1}{2}\left(Z_{c}+\frac{b}{d} N_{c}\right) \tilde{r}_{C, y} \\
M & =M_{c}+\frac{1}{2}\left(Z_{c}-\frac{b}{d} N_{c}\right) \tilde{r}_{C, x} .
\end{aligned}
$$

The disturbance value is unknown and its effect may be compensated by resorting to several control laws well known in the literature, in the following a PID is used.

\section{Center of mass estimation}

In case a simple PD control is used for pitch and roll control, a steady state error occurs. This effect can be counteracted linking the roll-pitch error to a proper integral estimate of the center of mass position according to:

$$
\left[\begin{array}{l}
\dot{\hat{r}}_{C, x} \\
\dot{\hat{r}}_{C, y}
\end{array}\right]=-k_{r_{C}}\left[\begin{array}{l}
\theta_{d}-\theta \\
\varphi_{d}-\varphi
\end{array}\right]
$$

to be used in eq. (5).

The stability analysis, not reported here for lack of space, has been discussed in [22]. 


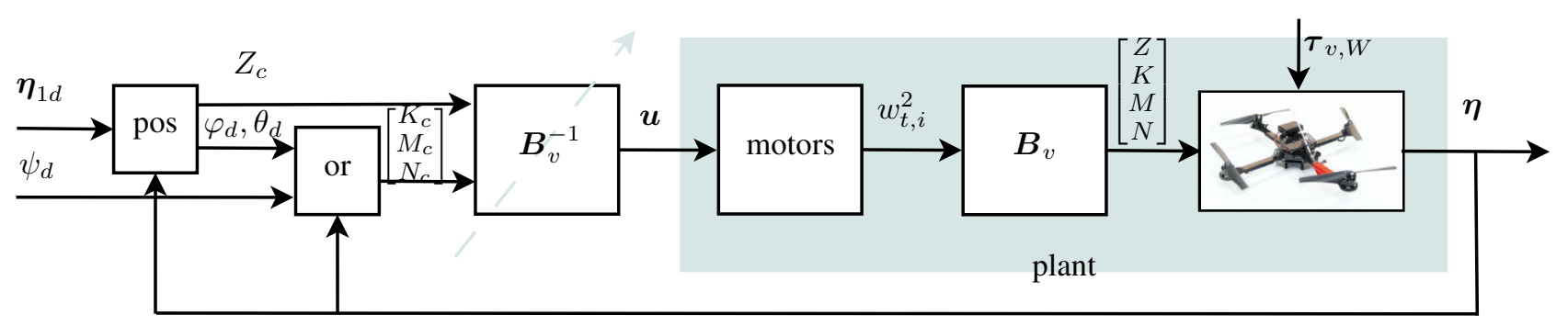

Fig. 2. Sketch of the control loop

\section{EXPERIMENTAL RESULTS}

The adaptive controller presented in the previous Sections has been both validated via numerical case studies and through actual experiments. The numerical tests have been performed on the SwarmSimX simulation environment [27], a software simulator developed by the Max Planck Institute for Biological Cybernetics (MPI) to debug and tune the controller parameters. The corresponding results are not reported here for reasons of space.

The MPI facilities have also been used for the experimental validation. The employed aerial vehicle is an improved version of the $M K$-Quadro ${ }^{1}$ quadrotor. The TeleKyb framework [28] has been used both for the adaptive control development and experimental-flow management. The quadrotor is equipped with an Atmega1284p microcontroller and a GNU-Linux onboard computer running a ROS-based software $^{2}$. The microcontroller has direct access to the onboard Inertial Measurement Unit (IMU), consisting of a 3D accelerometer and a 3D gyroscope. As for the quadrotor position/orientation, we made use of an external infraredvision-based system with an accuracy of $0.001 \mathrm{~m}$ in position and $1 \mathrm{deg}$ in orientation at the sampling frequency of $120 \mathrm{~Hz}$. Roll and pitch angles and the attitude controller are executed at a sampling frequency of $500 \mathrm{~Hz}$. The position controller and all the remaining computations are run on the onboard computer at $120 \mathrm{~Hz}$.

In order to test the controller with the minimal set of parameters, we have implemented the sole reduced version, i.e., the version in (8), (9), (10) and (11).

The desired trajectory is shown in figure 3 and it is the same for all the results reported in this paper.

In a first set of tests, the controller is run with and without the adaptive action, i.e., by imposing null adaptive gains. In a second set of tests, the CoM is displaced by attaching a weight, unknown to the controller, along the $x$-fixed direction as shown in Figure 4. The new position of the CoM was not exactly known, but, as a rough estimate estimate, it resulted displaced of $\approx 2 \mathrm{~cm}$. The four case studies are summarized in table I.

The following gains have been used for all the four case studies: $\lambda_{z}=3, k_{p, z}=5.5, \lambda_{x y}=3, k_{p, x y}=3, k_{v, \varphi \theta \psi}=1$, $k_{p, \varphi \theta \psi}=1$. For the sole adaptive cases, i.e., cases $\mathrm{b}$ and $\mathrm{d}$, the adaptive gains were different from zero: $k_{\gamma, z}=1.5$, $k_{\gamma, x y}=1$ and $k_{r C}=0.1$.

\footnotetext{
${ }^{1}$ http://www.mikrokopter.de

${ }^{2}$ www.ros.org
}

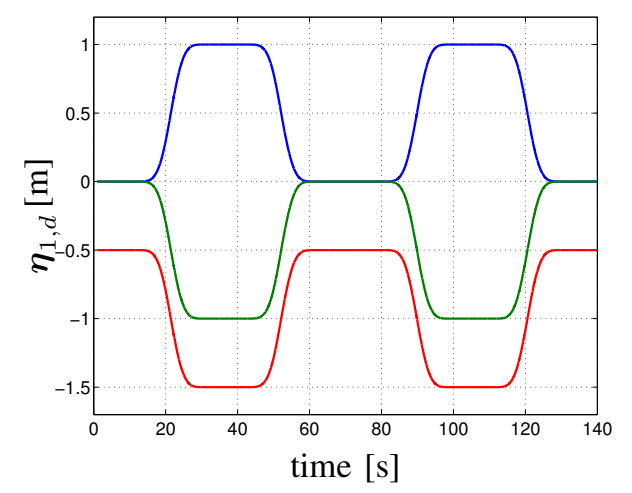

Fig. 3. Desired trajectory for the four experiments illustrated, blue denotes the inertial $x$ component, green the $y$ and red the $z$.

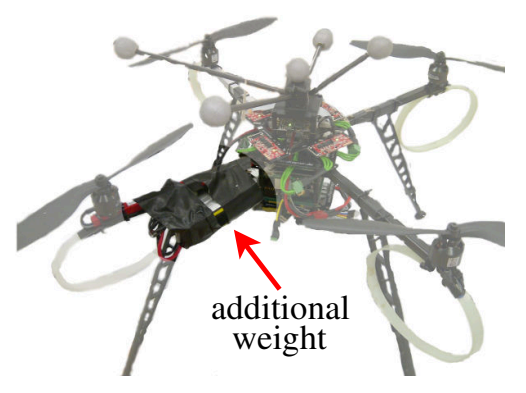

Fig. 4. mikrokopter with the unknown weight attached

Figure 5 reports the norm of the 3D position errors for the cases a) and b). It is worth noticing that the error is comparable during the transient, due to the intentional choice to adapt only with respect to the persistent terms, while it results considerably reduced for the adaptive version during the steady state. The error present in the non-adaptive version are mainly due to small external disturbances, unproper weight compensation as well as errors in the Vicon calibration.

Orientation errors, forces/moments at the vehicles and thrust for cases a) and b) are omitted to preserve space. It is interesting to discuss the cases c) and d), i.e., when an unknown wheight is attached to the vehicle. Figure 6 reports the norm of the 3D position errors. The same comments made for the cases a) and b) hold also in this case, but now the non-adaptive version exhibits larger errors while the adaptive one is able to compensate for the displaced center of mass.

It is also interesting to show the roll and pitch angles for the cases c) and d) in Figure 7. At steady state the adaptive 


\begin{tabular}{lll}
\hline case & weight & adaptive \\
\hline a) & no & no \\
b) & no & yes \\
c) & yes & no \\
d) & yes & yes \\
\hline
\end{tabular}

TABLE I

TYPE OF EXPERIMENTS

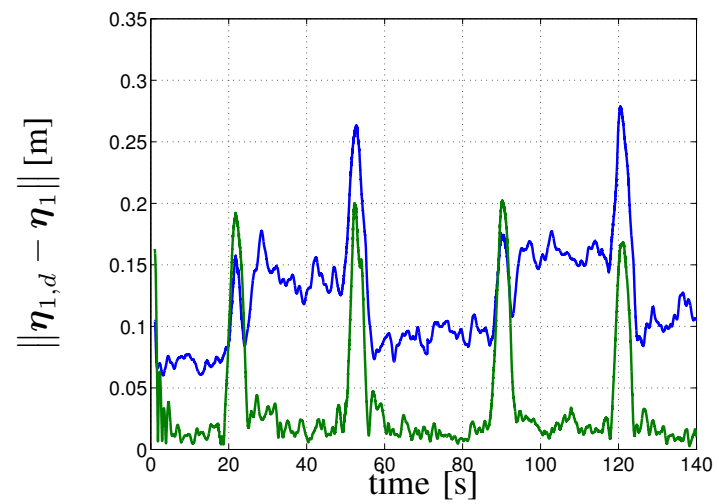

Fig. 5. Norm of the 3D position errors for the cases a) (in blue) and b) (in green)

controller compensates for the asymmetric CoM position by properly adapting the gain (and thus with null pitch), while the non adaptive case reaches a steady state with a non-zero pitch (and a corresponding non-zero position error).

Figure 8 reports the time history of the parameters for case d). It can be noticed that the parameter $\boldsymbol{r}_{C}$, i.e., the CoM estimation, is coherent with the rough estimate available. Figure 9 reports the generalized forces for the cases c) and d). It can be noticed that the improvement achieved in case d) is not due to an increased control effort.

Finally, a quantitative index has been used to verify the improvement in terms of position error, $i_{1}$, and the possible increase of control effort, $i_{2}$. By letting $n$ represent the number of samples of the experiment, index $i_{1}$ is defined as

$$
i_{1}=\frac{1}{n} \sum_{k=1}^{n}\left\|\boldsymbol{\eta}_{1, d}\left(t_{k}\right)-\boldsymbol{\eta}_{1}\left(t_{k}\right)\right\|
$$

while $i_{2}$ is defined as

$$
i_{2}=\frac{100}{U} \sum_{k=1}^{n} \sum_{j=1}^{4}\left|u_{j}\left(t_{k}\right)\right|
$$

i.e., normalized to 100 with respect to $U$, the maximum among the 4 case studies presented in this paper.

The result are shown in table II. It can be noticed that the errors are reduced without requiring an increase of control effort.

\section{CONCLUSIONS}

This paper presented the experimental validation of a new adaptive control law for quadrotors. For the system at hand, the disturbances were the vehicle weight, the center of mass position and the presence of a 6 DOFs external disturbance. The experiments, run at the Max Planck Institute facilities,

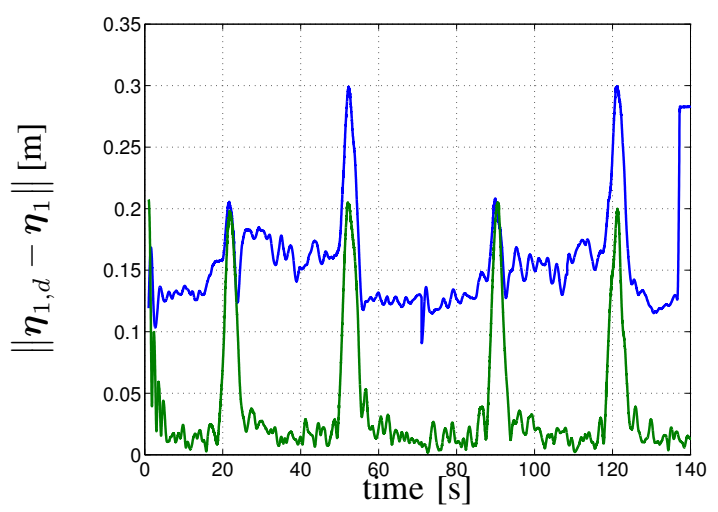

Fig. 6. Norm of the 3D position errors for the cases c) (in blue) and d) (in green)
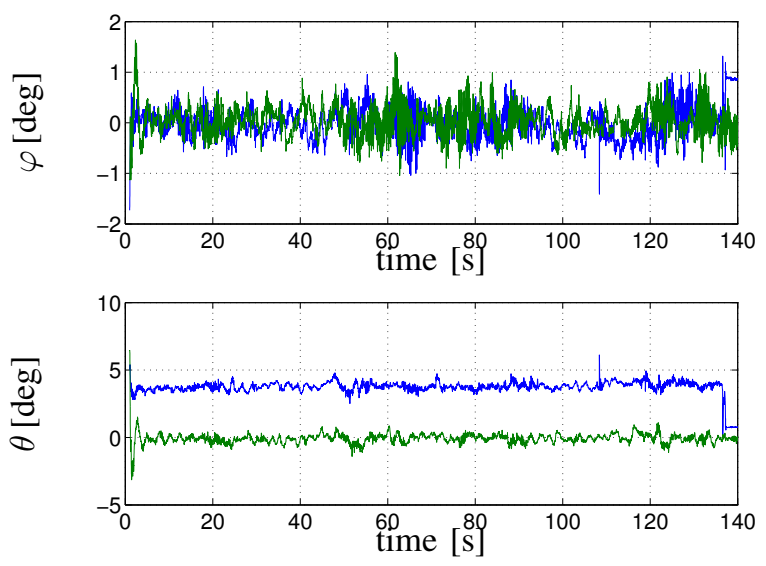

Fig. 7. Roll (top) and pitch (bottom) angles for the cases c) (in blue) and d) (in green)
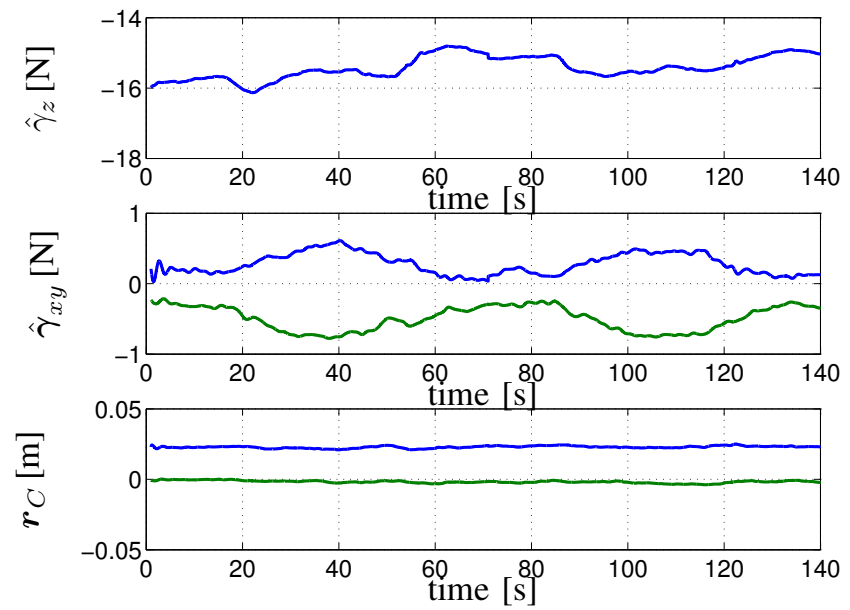

Fig. 8. Time history of the parametes for the case d). Top: parameter $\gamma_{z}$, center: parameter $\hat{\gamma}_{x y}$, bottom: parameter $\boldsymbol{r}_{C}$. It can be noticed that the center of mass estimation is coherent with the rough estimate done 

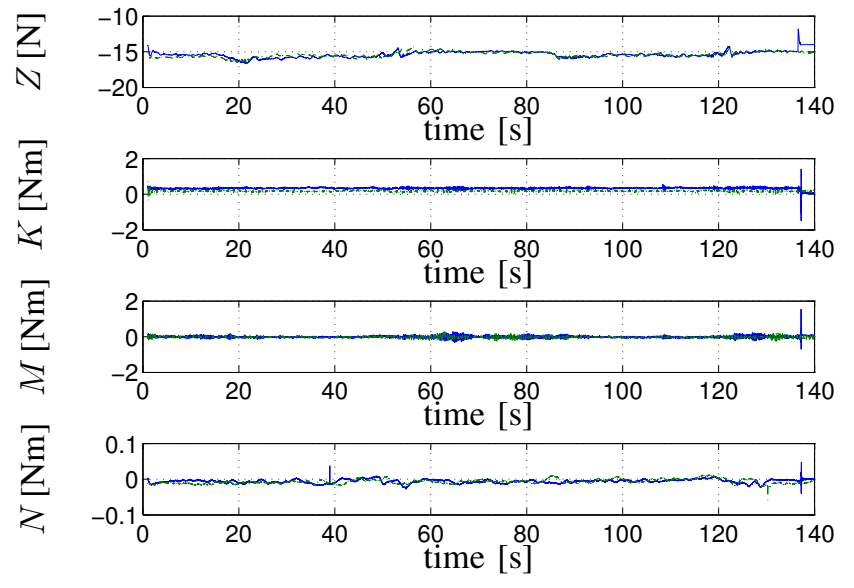

Fig. 9. Control forces for the cases c) and d)

\begin{tabular}{lll}
\hline case & $i_{1}[\mathrm{~cm}]$ & $i_{2}[-]$ \\
\hline a) & 12.4 & 86.2 \\
b) & 3.6 & 89.4 \\
c) & 15.7 & 99.7 \\
d) & 3.7 & 100.0 \\
\hline
\end{tabular}

TABLE II

ERROR AND EFFORT INDEXES

confirm the efficiency of the proposed controller. Reliability and robustness of MAV control will consistently be enhanced by using the proposed adaptive scheme, especially in presence of wind and during pick and place operations of small loads.

\section{ACKNOWLEDGEMENTS}

This research has received funding from the European Community's Seventh Framework Programme under grant agreement n. 287617 (IP project ARCAS - Aerial Robotics Cooperative Assembly system).

\section{REFERENCES}

[1] "Ascending Technology," in http://www. asctec.de/home-en.

[2] N. Michael, D. Mellinger, Q. Lindsey, and V. Kumar, "The GRASP multiple micro-UAV testbed," IEEE Robotics \& Automation Magazine, vol. 17 , no. 3 , pp. $56-65,2010$.

[3] A. Franchi, C. Secchi, M. Ryll, H. H. Bülthoff, and P. Robuffo Giordano, "Shared control: Balancing autonomy and human assistance with a group of quadrotor UAVs," IEEE Robotics \& Automation Magazine, Special Issue on Aerial Robotics and the Quadrotor Platform, vol. 19, no. 3, pp. 57-68, 2012.

[4] S. Weiss, D. Scaramuzza, and R. Siegwart, "Monocular-SLAM-based navigation for autonomous micro helicopters in GPS-denied environments," Journal of Field Robotics, vol. 28, no. 6, p. 854874, 2011.

[5] S. Shen, N. Michael, and V. Kumar, "Autonomous indoor 3D exploration with a micro-aerial vehicle," in 2012 IEEE Int. Conf. on Robotics and Automation, St. Paul, MN, May 2012, pp. 1050-4729.

[6] V. Grabe, H. H. Bülthoff, and P. Robuffo Giordano, "On-board velocity estimation and closed-loop control of a quadrotor UAV based on optical flow," in 2012 IEEE Int. Conf. on Robotics and Automation, St. Paul, MN, May 2012, pp. 491-497.

[7] J. Fink, N. Michael, S. Kim, and V. Kumar, "Planning and control for cooperative manipulation and transportation with aerial robots," The International Journal of Robotics Research, vol. 30, no. 3, pp. 324-334, 2011.

[8] R. Spica, A. Franchi, G. Oriolo, H. H. Bülthoff, and P. Robuffo Giordano, "Aerial grasping of a moving target with a quadrotor UAV," in 2012 IEEE/RSJ Int. Conf. on Intelligent Robots and Systems, Vilamoura, Portugal, Oct. 2012, pp. 4985-4992.
[9] A. Franchi, C. Masone, V. Grabe, M. Ryll, H. H. Bülthoff, and P. Robuffo Giordano, "Modeling and control of UAV bearingformations with bilateral high-level steering," The International Journal of Robotics Research, Special Issue on 3D Exploration, Mapping, and Surveillance, vol. 31, no. 12, pp. 1504-1525, 2012.

[10] P. Robuffo Giordano, A. Franchi, C. Secchi, and H. H. Bülthoff, "Bilateral teleoperation of groups of UAVs with decentralized connectivity maintenance," in 2011 Robotics: Science and Systems, Los Angeles, CA, Jun. 2011.

[11] N. Michael and V. Kumar, "Control of ensembles of aerial robots," Proceedings of the IEEE, vol. 99, no. 9, pp. 1587-1602, 2011.

[12] A. Franchi, C. Secchi, H. I. Son, H. H. Bülthoff, and P. Robuffo Giordano, "Bilateral teleoperation of groups of mobile robots with time-varying topology," IEEE Trans. on Robotics, vol. 28, no. 5, pp. 1019-1033, 2012.

[13] P. Castillo, R. Lozano, and A. Dzul, "Stabilization of a mini rotorcraft with four rotors," IEEE Control Systems Magazine, vol. 25, no. 6, pp. 45-55, 2005.

[14] M.-D. Hua, T. Hamel, P. Morin, and C. Samson, "A control approach for thrust-propelled underactuated vehicles and its application to VTOL drones," IEEE Trans. on Automatic Control, vol. 54, no. 8, pp. 1837-1853, 2009.

[15] S. Bouabdallah and R. Siegwart, "Backstepping and sliding-mode techniques applied to an indoor micro," in 2005 IEEE Int. Conf. on Robotics and Automation, May 2005, pp. 2247-2252.

[16] P. Adigbli, C. Gr, J. Mouret, and S. Doncieux, "Nonlinear attitude and position control of a micro quadrotor using sliding mode and backstepping techniques," in EMAV2007 - European Micro Air Vehicle Conference and Flight Competition, 2007.

[17] M. Fliess, J. Lévine, P. Martin, and P. Rouchon, "Flatness and defect of nonlinear systems: Introductory theory and examples," International Journal of Control, vol. 61, no. 6, pp. 1327-1361, 1995.

[18] M. Ryll, H. H. Bülthoff, and P. Robuffo Giordano, "First flight tests for a quadrotor UAV with tilting propellers," in 2013 IEEE Int. Conf. on Robotics and Automation, Karlsruhe, Germany, May 2013, pp. 295302.

[19] G. Antonelli, "On the use of adaptive/integral actions for 6-degrees-offreedom control of autonomous underwater vehicles," IEEE Journal of Oceanic Engineering, vol. 32, no. 2, pp. 300-312, April 2007.

[20] I. Palunko and R. Fierro, "Adaptive control of a quadrotor with dynamic changes in the center of gravity," in Proceedings 18th IFAC World Congress, vol. 18, no. 1, Milan, I, August 2011, pp. 2626-2631.

[21] I. Palunko, P. Cruz, and R. Fierro, "Agile load transportation. Safe and efficient load manipulation with aerial robots," IEEE Robotics \& Automation Magazine, vol. 19, no. 3, pp. 69-79, 2012.

[22] G. Antonelli, F. Arrichiello, S. Chiaverini, and P. Robuffo Giordano, "Adaptive trajectory tracking for quadrotor MAVs in presence of parameter uncertainties and external disturbances," in Proceedings 2013 IEEE/ASME International Conference on Advanced Intelligent Mechatronics, submitted, Wollongong, AU, July 2013.

[23] B. Siciliano, L. Sciavicco, L. Villani, and G. Oriolo, Robotics: modelling, planning and control. Springer Verlag, 2009.

[24] T. Fossen, Marine Control Systems: Guidance, Navigation and Control of Ships, Rigs and Underwater Vehicles. Trondheim, Norway: Marine Cybernetics, 2002.

[25] M. Gautier and W. Khalil, "Direct calculation of minimum set of inertial parameters of serial robots," IEEE Trans. on Automatic Control, vol. 6, no. 3, pp. 368-373, 1990.

[26] G. Antonelli, F. Caccavale, and P. Chiacchio, "A systematic procedure for the identification of dynamic parameters of robot manipulators," Robotica, vol. 17, no. 4, pp. 427-435, 1999.

[27] J. Lächele, A. Franchi, H. H. Bülthoff, and P. Robuffo Giordano, "SwarmSimX: Real-time simulation environment for multi-robot systems," in 3rd Int. Conf. on Simulation, Modeling, and Programming for Autonomous Robots, Tsukuba, Japan, Nov. 2012.

[28] V. Grabe, M. Riedel, H. H. Bülthoff, P. Robuffo Giordano, and A. Franchi, "The TeleKyb framework for a modular and extendible ROS-based quadrotor control," in 6th European Conference on Mobile Robots, Barcelona, Spain, Sep. 2013. 